\title{
Institutional Board Review for Clinical Investigations on Inflammatory Bowel Diseases: A Single-Center Study
}

\author{
Sinyoung Park ${ }^{1}$, Yang Hee Noh ${ }^{1}$, Sun Young Rha ${ }^{1,2}$, Won Ho Kim ${ }^{3}$, Jae Hee Cheon ${ }^{3}$ \\ Human research Protection Center, Severance Hospital, Yonsei University Health System ${ }^{1}$, Seoul, Department of Internal Medicine and Institute \\ of Oncology ${ }^{2}$, Department of Internal Medicine and Institute of Gastroenterology, Yonsei University College of Medicine, Seoul, Korea
}

Background/Aims: The growing volume and the diversity of clinical research has led to related laws and regulations as well as the Institutional Review Board (IRB) approval process becoming more stringent. To conduct clinical research efficiently and while following regulations, information about the IRB approval process and feedback is important for investigators. This has yet to be studied. Methods: We included 381 gastrointestinal disease research proposals (79 with inflammatory bowel disease [IBD], and 302 with non-IBD) reviewed by the IRB of Severance Hospital between January 2009 and December 2013. We retrospectively analyzed research characteristics including research risk levels, results of initial reviews, frequencies of continuing review, numbers of IRB comments, frequencies of IRB comments, and durations from submission to approval. Results: Investigators' decisions on risk level were higher in the IBD group than in the non-IBD group $(P<0.05)$. Results of initial reviews, frequencies of continuing reviews, the numbers of IRB review comments, and durations from submission to approval were not different between the two groups, but IRB decisions on risk level were higher in the IBD group $(P<0.05)$. In subgroup analysis, the number of IRB comments from initial review on informed consent forms and procedures as well were quest of more information were significantly higher in the IBD group than in the non-IBD group $(P<0.001$ and 0.01 , respectively). Conclusions: In Korea, rare diseases such as IBD require more information for the IRB process due to their distinct characteristics. IBD researchers should develop research protocols more carefully and make their research as subject-friendly as possible. (Intest Res 2015;13:274-281)

Key Words: Inflammatory bowel diseases; Institutional review board; Institutional review board recommendation

\section{INTRODUCTION}

Inflammatory bowel disease (IBD), which mainly consists of UC, CD, and intestinal Behçet's disease, involves chronic inflammation of the gastrointestinal (GI) tract with unknown etiology, and is characterized by a remitting and

Received August 16, 2014. Revised November 5, 2014.

Accepted November 5, 2014.

Correspondence to Jae Hee Cheon, Department of Internal Medicine, Yonsei University College of Medicine, 50-1 Yonsei-ro, Seodaemun-gu, Seoul 120-752, Korea. Tel: +82-2-2228-1990, Fax: +82-2-393-6884, E-mail: geniushee@yuhs.ac

Financial support: This work was supported by Basic Science Research Program through the National Research Foundation of Korea funded by the Ministry of Science, ICT \& Future Planning (NRF-2013R1A2A2A01067123) and grants of the Korean Health Technology RetD Project, Ministry of Health \& Welfare, Republic of Korea (A120176 and HI13C1345). Conflict of interest: None. relapsing course. Since the incidence and prevalence of CD and UC show a continuously increasing trend in Korea and other Asian countries, medical scientists have grown more interested in these diseases. ${ }^{1-3}$ With the increasing prevalence in IBD and advances in the field of molecular biology, an increasing number of studies have investigated IBD, and this trend is not just limited to IBD. Expanded infrastructure and clinical research capabilities have contributed to the advancement of clinical studies investigating various kinds of disease during the last two decades. ${ }^{4}$ After the second world war, medical ethics expanded to ethical principles for clinical research, based on the Nuremberg Code published in 1947 and the Declaration of Helsinki published in 1964, and the necessity of Institutional Review Board (IRB) approval was first introduced. ${ }^{5}$ All research using human subjects, such as clinical trials for new drug/medical device approval, in addi-

\footnotetext{
๑ Copyright 2015. Korean Association for the Study of Intestinal Diseases. All rights reserved.

This is an Open Access article distributed under the terms of the Creative Commons Attribution Non-Commercial License (http://creativecommons.org/licenses/by-nc/4.0)

which permits unrestricted non-commercial use, distribution, and reproduction in any medium, provided the original work is properly cited.
} 
tion to human biospecimen research, must be reviewed and approved by an IRB. The IRB process serves an important and basic role in the protection of clinical research and human research subjects. However, IRB review and approval can sometimes be burdensome for investigators. Thus, investigators are committed to conducting high quality clinical research by designing a clinical study protocol in a time efficient-manner and in accordance with laws and regulations, and to going through the IRB review process. Investigators studying rare diseases have difficulty gaining approval from IRBs or other government agencies (Food and Drug Administration etc.), and this seems to be attributed to the lack of information and knowledge on these diseases. Close cooperation between experts with experience in rare disorders and investigators can help in successfully navigating the IRB approval process. ${ }^{6}$

IBD is considered a rare and intractable disorder in Korea. Since IBD is a chronic disorder, unlike common GI diseases, some considerations need to be taken into account when gaining IRB approval according to reviewers' or sponsors' experience, study protocol, and IRB submission and review. A few studies have been performed on the IRB process so far. ${ }^{7}$ In the 1970s, previous studies mainly investigated the structure of IRB, workforce, work intensity, and the scope of studies subject to review. ${ }^{8-11}$ IRB review has been found to focus on obtaining written informed consent and agreement. ${ }^{12}$ Subsequently, studies on deviations from IRB review results by institutions were carried out in relation to reviews for multi-institutional clinical trials, and differences in evaluation outcomes and IRB comments, even with the same study design, were statistically significant. ${ }^{13-15}$ However, no studies have suggested guidelines for study protocols or for investigators receiving reviews through analysis of IRB evaluation outcomes and comments, or have been performed on the characteristics of the IRB's review of research on rare diseases such as IBD in Korea.

Therefore, this study aims to identify differences between IBD and non-IBD GI studies by analyzing factors such as review types, evaluation outcomes, approval processing times, and comments.

\section{METHODS}

\section{Materials and Methods}

This study involved 381 GI disease research proposals reviewed by the IRB of Severance Hospital, Yonsei University Health System, between January 1, 2009 and December
31, 2013. To collect accurate data, we included studies performed by principal investigators in the Division of Gastroenterology, and excluded studies performed by investigators from other departments such as General Surgery or Oncology. Studies on CD, UC, and intestinal Behçet's disease, and studies simultaneously investigating IBD and other GI diseases were classified into the IBD group.

We examined types of clinical studies (investigator-initiated trials [IITs]), sponsor-initiated trials [SITs]), study phases, results of initial reviews, and durations from submission to approval based on the IRB database managed by the Human Research Protection Center, Severance Hospital, Korea. We also analyzed research risk levels determined by investigators and IRBs, frequencies of continuing review, and IRB comments in IRB review request forms and the minutes of each study. Study risk was divided into four levels, as used by the IRB of Severance Hospital. The levels of risk included: (1) Level 1: no greater than minimal risk; (2) Level 2: minor increase over minimal risk; (3) Level 3: moderate risk with a medium to high probability of a moderate-severity event; and (4) Level 4: high risk greater than a moderate risk study due to the increased probability for generating irreversible morbidity or serious adverse events.

IRB comments were classified into eight categories including: (1) risk level and data/safety monitoring plan, frequency of continuing review, (2) study design, (3) statistics, (4) informed consent form and procedure, (5) research resources (research grant, researchers etc.), (6) subject protection, (7) minor modification, and (8) more information needed based on the classification of Lidz CW et al. ${ }^{16}$ Since this study did not involve human subjects, the study was conducted without IRB approval.

\section{Statistical Analysis}

Quantitative variables were presented as mean $\pm S D$, while non-quantitative variables were presented as numerical values (percentage). Excluding retrospective studies, the results of initial review according to diseases, approval duration, and comments were analyzed, and variables were compared using Student's $t$-test, Fisher's exact test, and the chi-square test. The agreement between risk levels decided by investigators and IRB in IBD and non-IBD studies was assessed using a Kappa value, and differences between paired proportions were determined with McNemar's test. Agreement of Kappa values ranging from 0.0 to 1.0 was defined as follows: 0.00-0.20: slight agreement; 0.2-0.4: fair agreement; 0.4-0.6: moderate agreement; 0.6-0.8: substantial agreement; and 
0.8-1.0: almost perfect agreement. $P$-values of $<0.05$ were considered statistically significant. All statistical analyses were conducted using PASW Statistics 18.0 (ver. 18.0; IBM Co., Armonk, NY, USA).

\section{RESULTS}

\section{Baseline Characteristics of Studies}

Of all GI disease research proposals reviewed by the IRB of Severance Hospital between January 2009 and December 2013, 79 were IBD studies and (20.7\%) and 302 were non-IBD GI studies (79.3\%). Of all IBD studies, 27 were IITs (34.2\%) and 52 were SITs (65.8\%). Of all non-IBD GI studies, 104 were SITs (34.4\%) and 198 were SITs (65.6\%). No statistically significant difference was found between the two groups $(P=0.965)$. According to study phase, IBD studies consisted of a phase I trial (1.3\%), 10 phase II trials (12.7\%), 11 phase III trials (13.9\%), and a phase IV trial (1.3\%). Meanwhile, there were three post-marketing survey studies (3.8\%), 32 clinical trials including registry, cohort, human biospecimen, or survey studies (40.5\%), and 21 retrospective studies (26.6\%). There was neither a phase I/II clinical trial nor a clinical trial for medical devices. According to the types of study design, a statistically significant difference was not detected between the two groups $(P=0.238)$. According to initial review data, the number of reviewed IBD studies increase annually, and then decreased in 2013 compared to 2012. No significant diff-

Table 1. Baseline Characteristics of Clinical Research Between IBD and Non-IBD Gastrointestinal Studies

\begin{tabular}{|c|c|c|c|c|}
\hline Characteristics & Total $(n=381)$ & IBD studies $(n=79)$ & Non-IBD studies $(n=302)$ & $P$-value \\
\hline Study & & & & $0.965^{*}$ \\
\hline SIT & $131(34.4)$ & $27(34.2)$ & $104(34.4)$ & \\
\hline Phase & & & & $0.238^{+}$ \\
\hline Phase I & $2(0.5)$ & $1(1.3)$ & $1(0.5)$ & \\
\hline Phase II & $40(10.5)$ & $10(12.7)$ & $30(15.5)$ & \\
\hline Phase III & $51(13.4)$ & $11(13.9)$ & $40(20.6)$ & \\
\hline Phase IV & $34(8.9)$ & $1(1.3)$ & $33(17.0)$ & \\
\hline Medical device clinical trial & $3(0.8)$ & $0(0.0)$ & $3(1.5)$ & \\
\hline No. of study reviewed & & & & $0.211^{+}$ \\
\hline 2009 & $56(14.7)$ & $5(6.3)$ & $51(16.9)$ & \\
\hline 2010 & $76(20.0)$ & $15(19.0)$ & $61(20.2)$ & \\
\hline 2011 & $66(17.3)$ & $21(26.6)$ & 45 (14.9) & \\
\hline 2012 & $99(26.0)$ & $21(26.6)$ & $78(25.8)$ & \\
\hline 2013 & $84(22.0)$ & $17(21.5)$ & $67(22.2)$ & \\
\hline Risk level submitted by investigator & & & & $0.000^{*}$ \\
\hline Level 1 & $273(76.5)$ & $59(76.6)$ & $214(76.4)$ & \\
\hline
\end{tabular}

Values are presented as $n(\%)$.

*Fisher's exact test.

${ }^{+}$Chi-squared test.

SIT, sponsor initiated trial; IIT, investigator initiated trial; PMS, post marketing survey. 
erence was found between the two groups $(P=0.211)$ (Table 1).

\section{IRB Review Results}

\section{1) Results of Initial Review}

This study analyzed 299 prospective trials by categorizing them into approved, conditionally approved, approved with modification, deferred, or rejected classes. In the comparison of results of initial review between the IBD and non-IBD GI groups, no significant difference was found between the two groups $(P=0.368)$. With respect to the number of IRB comments, there were $3.9 \pm 4.0$ comments in IBD studies and $3.5 \pm 4$.1 comments in non-IBD GI studies. Even though the number of comments was higher in IBD studies, there was no significant difference between the two groups $(P=0.580)$. Time from initial review to approval took $28.2 \pm 41.8$ days in IBD studies and 31.6 \pm 48.3 days in non-IBD studies. Despite the longer duration of approval process in non-IBD GI studies, no significant difference was observed $(P=0.662)$ (Table 2).

\section{2) Risk Level and Frequency of Continuing Review}

Risk levels determined by investigators and IRB were compared in 277 of the 299 prospective studies, excluding 22 studies without risk level. IRB decisions on risk level were higher in $15.8 \%$ of IBD studies and $27.2 \%$ of non-IBD GI studies. IRB decisions on risk level were significantly higher in the non-IBD group (Table 3$)(P<0.001)$. In analysis of agreement between risk levels decided by investigators and IRB, both groups showed moderate agreement or higher. The degree of agreement was higher in the IBD group than in the non-IBD GI group (kappa 0.65 vs. 0.50$).{ }^{17}$ IRB frequency of continuing review was determined by considering the characteristics and risk levels of all reviewed studies. In the IBD group, intervals for interim reviews were every 12 months in 48 studies (82.8\%), every 6 months in three studies (5.2\%), and exempt in seven studies (12.1\%). The frequency of continuing review was determined in all studies during the initial IRB review. In the non-IBD GI group, intervals for interim reviews were every 12 months in 201 studies (83.4\%), every 6 months in 12 studies (5.0\%), and exempt in 26 studies $(10.8 \%)$. The frequency of continuing review was not determined in two studies $(0.8 \%)$. There was no significant difference between the two groups $(P=0.906)$.

Table 2. Result of Institutional Review Board (IRB) Review Between IBD and Non-IBD Gastrointestinal Studies

\begin{tabular}{|c|c|c|c|c|}
\hline Results & Total $(n=299)$ & IBD studies $(n=58)$ & Non-IBD studies $(n=241)$ & $P$-value \\
\hline Result of initial review & & & & $0.368^{*}$ \\
\hline Approved & $130(43.5)$ & $30(51.7)$ & $100(41.5)$ & \\
\hline Approved with modification & $122(40.8)$ & $20(34.5)$ & $102(42.3)$ & \\
\hline Deferred & $47(15.7)$ & $8(13.8)$ & $39(16.2)$ & \\
\hline Disapproved & $0(0.0)$ & $0(0.0)$ & $0(0.0)$ & \\
\hline Tabled & $0(0.0)$ & $0(0.0)$ & $0(0.0)$ & \\
\hline Risk Level & & & & $0.001^{*}$ \\
\hline Level 1 & $147(49.2)$ & $33(56.9)$ & $114(47.3)$ & \\
\hline Level 2 & $101(33.8)$ & $13(22.4)$ & $88(36.5)$ & \\
\hline Level 3 & $44(14.7)$ & $7(12.1)$ & $37(15.4)$ & \\
\hline Level 4 & $7(2.3)$ & $5(8.6)$ & $2(0.8)$ & \\
\hline Frequency of continuing review & & & & $0.906^{*}$ \\
\hline Every 12 mo & 249 (83.3) & $48(82.8)$ & $201(83.4)$ & \\
\hline Every 6 mo & $15(5.0)$ & $3(5.2)$ & $12(5.0)$ & \\
\hline Exempt & $33(11.0)$ & $7(12.1)$ & $26(10.8)$ & \\
\hline Pending & $2(0.7)$ & $0(0.0)$ & $2(0.8)$ & \\
\hline Number of IRB review comment & $3.6 \pm 4.2$ & $3.9 \pm 4.0$ & $3.5 \pm 4.1$ & $0.580^{\dagger}$ \\
\hline Duration from submission to approval (days) & $30.5 \pm 47.0$ & $28.2 \pm 41.8$ & $31.6 \pm 48.3$ & $0.662^{+}$ \\
\hline
\end{tabular}

Values are presented as $n(\%)$ or mean \pm SD.

*Fisher's exact test.

${ }^{\dagger}$ Independent $t$-test. 
Table 3. Risk Level of Study $\left(n=277^{*}\right)$

\begin{tabular}{|c|c|c|c|c|c|c|}
\hline \multirow{2}{*}{ IRB decision } & \multicolumn{5}{|c|}{ Investigator } & \multirow{2}{*}{$P$-value } \\
\hline & Level 1 & Level 2 & Level 3 & Level 4 & Total & \\
\hline IBD study & & & & & & $0.050^{+}$ \\
\hline Level 1 & $33(57.9)$ & $0(0.0)$ & $0(0.0)$ & $0(0.0)$ & $33(57.9)$ & \\
\hline Level 2 & $4(7.0)$ & $8(14.0)$ & $0(0.0)$ & $0(0.0)$ & $12(21.1)$ & \\
\hline Level 3 & $2(3.5)$ & $2(3.5)$ & $1(1.8)$ & $2(3.5)$ & $7(12.3)$ & \\
\hline Level 4 & $0(0.0)$ & $1(1.8)$ & $0(0.0)$ & $4(7.0)$ & $5(8.8)$ & \\
\hline Total & $39(68.4)$ & $11(19.3)$ & $1(1.8)$ & $6(10.5)$ & 57 & \\
\hline Non-IBD GI study & & & & & & $<0.001^{+}$ \\
\hline Level 1 & $106(48.2)$ & $0(0.0)$ & $0(0.0)$ & $0(0.0)$ & $106(48.2)$ & \\
\hline Level 2 & $36(16.4)$ & $43(19.6)$ & $2(1.0)$ & $0(0.0)$ & $81(36.8)$ & \\
\hline Level 3 & $11(5.0)$ & $11(5.0)$ & $9(4.1)$ & $0(0.0)$ & $31(14.1)$ & \\
\hline Level 4 & $1(0.4)$ & $1(0.4)$ & $0(0.0)$ & $0(0.0)$ & $2(0.9)$ & \\
\hline Total & $154(70.0)$ & $55(25.0)$ & $11(5.0)$ & $0(0.0)$ & 220 & \\
\hline
\end{tabular}

Values are presented as $n(\%)$ or mean \pm SD.

Note that percentages of study that risk level elevated are 27.2\% for IBD group and 15.8\% for non-IBD group, presented as bold section.

*Decision on risk level has been performed since 2010. The number of decision on risk level was 277 since 2010.

${ }^{+}$McNemar-Bowker test.

IRB, Institutional Review Board; GI, gastrointestinal.

Table 4. Comparison of IRB Comments Between IBD Research Group and Non-IBD Gastrointestinal Disease Research Group

\begin{tabular}{lccc}
\hline \multicolumn{1}{c}{ Variables } & IBD $(\mathbf{n}=\mathbf{5 8})$ & Non-IBD $(\mathbf{n}=\mathbf{2 4 1})$ & $\boldsymbol{P}_{\text {-value }}{ }^{*}$ \\
\hline Risk level/DSMP & $0.26 \pm 0.44$ & $0.52 \pm 0.65$ & 0.680 \\
Study design & $0.43 \pm 0.94$ & $0.63 \pm 1.06$ & 0.420 \\
Statistics & $0.09 \pm 0.42$ & $0.36 \pm 0.61$ & 0.040 \\
Informed consent form and procedure & $3.96 \pm 3.02$ & $2.30 \pm 2.17$ & $<0.001$ \\
Research resources & $0.13 \pm 0.34$ & $0.40 \pm 0.58$ & 0.030 \\
Subject protection & $0.43 \pm 0.79$ & $0.22 \pm 0.46$ & 0.080 \\
Minor modification & $0.48 \pm 0.67$ & $0.67 \pm 0.82$ & 0.300 \\
Need more information & $0.30 \pm 0.56$ & $0.09 \pm 0.31$ & 0.010 \\
\hline
\end{tabular}

Values are presented as mean \pm SD.

*Independent $t$-test.

IRB, Institutional Review Board; DSMP, data and safety monitoring plan.

\section{IRB Review Comments}

Comments suggested in the IRB's initial review were analyzed. Table 4 shows the comparison of IRB comments between the IBD and non-IBD GI groups. The number of comments on risk level, study design, statistics, research resources, and minor modification was higher in the nonIBD GI group. The mean number of comments on "informed consent form and procedure" $(P<0.001)$ and "need more information" $(P=0.01)$ was significantly higher in the IBD group.

\section{DISCUSSION}

To conduct clinical trials in accordance with the principles of the Good Clinical Practice (GCP) guidelines, investigators are required improve the quality of their studies based on overall evaluation and discussion of major IRB comments, causes of delayed review, and others from the IRB review process after developing a study protocol. This study aimed to identify beneficial points to consider when preparing an IRB research protocol by comparing types of IRB research, 
review results, approval processing times, comments and so on between IBD and non-IBD GI studies. SITs accounted for $34.2 \%$ and $34.4 \%$ of all studies in the IBD and non-IBD GI groups, respectively, showing no difference between the two groups.

The percentage of SITs was similar in the two groups at approximately $34 \%$. Since a clinical trial needed for a marketed product for every new drug candidate is carried out longterm, there was no significant increase in absolute number. The lower proportion of IITs for new drug approval is thought to be attributable to time and economic constraints resulting from the verification of adaptability and efficacy, combined modality, and others. Moreover, the greater percentage of IITs for purposes other than new drug approval is probably attributable to study materials obtained for clinical practice and a wide range of experimental fields.

Phase III trials account for a large percentage of all clinical trial phases. Among all types of study design, "others" including registry, cohort, human biospecimen, and survey studies accounted for the largest percentage of all categories. There was no significant difference in study phase between the two groups, because the proportion of other clinical trials is large as studies conducted for purposes other than drug development are more convenient and various. Since human subject research should be performed after gaining IRB approval as stated in law after the enforcement of the wholly amended Bioethics and Safety Act on February 2, 2013, the number of clinical trials classified as "others" is forecasted to grow.

As the types and number of clinical studies have increased, related laws and regulations are being reinforced. Furthermore, the scope of IRB has been expanded as the numbers of studies to be reviewed have increased. The numbers of studies on GI diseases are also increasing. The percentages of IBD and non-IBD GI studies were increased by $40 \%$ and $31.4 \%$ from 2009 to 2013, respectively. The increased number of IBD studies to be reviewed in recent years is thought to be attributable to increased prevalence and interest in IBD by Korean investigators.

In terms of levels of risk, the IBD group had more studies classified as level 4 (highest risk category) by the investigators themselves compared to the non-IBD GI group. This outcome most likely reflects the nature of chronic inflammation of the intestines of unknown cause that persistently lowers the quality of life in patients and increases the incidence of complications such as enterostenosis, fistula, and colon cancer. $^{18-20}$ Taking into account the fact that the age of onset is the $10 \mathrm{~s}-20 \mathrm{~s},{ }^{21}$ investigators seem to consider IBD studies as high-risk. In the comparison between IRB's and investiga- tor's decisions on risk level, both investigators and IRB had a tendency to consider IBD studies as high-risk. The number of studies with an elevated level of risk was higher in nonIBD GI studies compared to IBD studies (27.2\% vs. 15.8\%). Thus, investigators were found to evaluate the risk level of their IBD studies more strictly. To make IRB review more appropriately without bias, investigators must provide measures to minimize risk and a data/safety monitoring plan in their protocols when applying for IRB review.

No significant difference was found in the approval rate of initial review and duration from submission to first approval between the two groups. This is because IRB approval varies depending on study protocols and variation is considerable. The Standard Operating Procedure (SOP) is to document policies and procedures including application, approval, and notification for IRB review of research, and the due date for submission of answers to reviewers' comments. This seems to be the reason why there was no difference in duration to approval between the two groups. However, the IRB process takes about a month to review a new protocol, and this remains a problem. We suggest that IRB administrators should sufficiently reflect investigator's answers to IRB review comments as modifications and additional information in the pre-review process prior to the second review in order to shorten duration to approval.

There was no difference in IRB frequency of continuing review between the two groups. Frequency of continuing review is generally determined by considering study design, characteristics of subjects, and the risk-benefit ratio. Study protocols are typically reviewed on a continual basis at an interval of one year, and intervals of continuing review are shortened to every 3 or 6 months depending on the degree of risk as determined by the IRB. No significant difference was detected in the frequency of continuing review between the two groups. We consider this outcome as the result of the IRB determining the frequency of continuing review as 12 months in most protocols, and the intervals of continuing review have not been shortened in many studies with a risk level of 4.

In the comparison of the number of initial IRB comments between IBD and non-IBD GI studies, no significant difference was found between the two groups. Determining significant difference can be difficult for the number of comments, since a single comment in a protocol may point out a major error, while several comments in a protocol may be minor, requiring minimal modifications. According to the types of IRB comments, informed consent forms and procedures have been most commonly pointed out in IBD 
studies, followed by requests for additional information. The mean number of other comments was higher in non-IBD GI studies, and this needs to be noted since patients with highly prevalent diseases are more likely to assess a variety of information through general knowledge, mass media, and others compared to those with a disease with a low prevalence. Patients with relatively less information need to be fully informed with detailed explanations prior to participation in the study. Thus, the informed consent form should include a detailed and clear explanation of the research when investigators apply for IRB review. In addition, informed consent should be voluntarily obtained and documented by the use of a written consent form by performing prior informed consent procedure in accordance with the principles of GCP.

Ideal reviews could be performed if the IRB Committee consists of members with competence in special areas to assist in reviewing key issues. However, it is practically impossible to invite experts in all fields of study as IRB reviewers. For this reason, investigators should write their protocols in detail when applying for IRB review, in order to minimize IRB review opinions on study design and background. According to the results of our analysis, IBD studies had more comments requesting additional information compared to non-IBD GI studies. Detailed information about diseases should be also provided in research documents. Furthermore, the use of pre-review of IRB protocols can shorten approval processing times and increase approval rates.

There are several limitations to note in the present study. This investigation was a single-center retrospective study with a relatively small sample size. Deviation may occur according to the specificity of investigators conducting studies on IBD. Moreover, deviation may be present depending on factors such as IRB reviewers' experience and preferences of the IRB committee. Therefore, the results from this survey cannot be generalized. In addition, the effects of external factors such as committee members and changes in the IRB SOPs have not been considered in the annual analyses.

In conclusion, IBD studies were not significantly different to GI disease studies in terms of duration from submission to approval or approval rate. Nevertheless, appropriate decisions on risk level and preparation for protection measures are still crucial to the protection of human subjects. Furthermore, IBD investigators can reduce the burdens of the IRB approval process by developing research protocols and subject information sheets with detailed information and descriptions.

\section{REFERENCES}

1. Yang SK, Yun S, Kim JH, et al. Epidemiology of inflammatory bowel disease in the Songpa-Kangdong district, Seoul, Korea, 1986-2005: a KASID study. Inflamm Bowel Dis 2008;14:542549.

2. Thia KT, Loftus EV, Jr., Sandborn WJ, Yang SK. An update on the epidemiology of inflammatory bowel disease in Asia. Am J Gastroenterol 2008;103:3167-3182.

3. Park SJ, Kim WH, Cheon JH. Clinical characteristics and treatment of inflammatory bowel disease: a comparison of Eastern and Western perspectives. World J Gastroenterol 2014;20:11525-11537.

4. Woodward B. Challenges to human subject protections in US medical research. JAMA 1999;282:1947-1952.

5. World Medical Association Inc. Declaration of Helsinki. Ethical principles for medical research involving human subjects. J Indian Med Assoc 2009;107:403-405.

6. Griggs RC, Batshaw M, Dunkle M, et al. Clinical research for rare disease: opportunities, challenges, and solutions. Mol Genet Metab 2009;96:20-26.

7. Cheon JH, Kim YS, Ye BD, et al. Crohn's Disease Clinical Network and Cohort (CONNECT) study: the first step toward nationwide multicenter research of Crohn's disease in Korea. Intest Res 2014;12:173-175.

8. Barber B, Lally JJ, Makarushka JL, Sullivan D. Research on human subjects: problems of social control in medical experimentation. New York: Russell Sage Foundation, 1973.

9. Bartlett EE. International analysis of institutional review boards registered with the U.S. Office for human research protection. J Empir Res Hum Res Ethics 2008;3:49-56.

10. Bell J, Whiton J, Connelly S [Report]. Evaluation of NIH implementation of Section 491 of the Public Health Service Act, mandating a program of protection for research subjects (USA). Washington, D.C.: U.S. Department of Health and Human Services, 1998.

11. Catania JA, Lo B, Wolf LE, et al. Survey of U.S. human research protection organizations: workload and membership. J Empir Res Hum Res Ethics 2008;3:57-69.

12. Sansone RA, McDonald S, Hanley P, Sellbom M, Gaither GA. The stipulations of one institutional review board: a five year review. J Med Ethics 2004;30:308-310.

13. Shah S, Whittle A, Wilfond B, Gensler G, Wendler D. How do institutional review boards apply the federal risk and benefit standards for pediatric research? JAMA 2004;291:476-482.

14. McWilliams R, Hoover-Fong J, Hamosh A, Beck S, Beaty T, Cutting G. Problematic variation in local institutional review of a multicenter genetic epidemiology study. JAMA 2003;290:360366. 
15. Burman W, Breese P, Weis S, Bock N, Bernardo J, Vernon A; Tuberculosis Trials Consortium. The effects of local review on informed consent documents from a multicenter clinical trials consortium. Control Clin Trials 2003;24:245-255.

16. Lidz CW, Appelbaum PS, Arnold R, et al. How closely do institutional review boards follow the common rule? Acad Med 2012;87:969-974.

17. Altman DG. Practical statistics for medical research. London: Chapman \& Hall, 1991.

18. Bosani M, Ardizzone S, Porro GB. Biologic targeting in the treatment of inflammatory bowel diseases. Biologics 2009;3:77-97.
19. Kuhbacher T, Folsch UR. Practical guidelines for the treatment of inflammatory bowel disease. World J Gastroenterol 2007;13:1149-1155.

20. Jeon HH, Lee HJ, Jang HW, et al. Clinical outcomes and predictive factors in oral corticosteroid-refractory active ulcerative colitis. World J Gastroenterol 2013;19:265-273.

21. Johnston RD, Logan RF. What is the peak age for onset of IBD? Inflamm Bowel Dis 2008;14(Suppl 2):S4-S5. 